\title{
A NOTE ON THE ACTION SPECTRUM OF HUMAN ROD VISION
}

\author{
M. ALPERN \\ Vision Research Laboratory, W. K. Kellogg Eye Center, University of Michigan, \\ Ann Arbor, MI 48105, U.S.A.
}

(Received 24 July 1986; in revised form 5 January 1987)

\begin{abstract}
A template representing the spectral distribution of the absorption coefficients of human thodospin was fitted to each of 59 individual action spectra of human rod vision (from one of three populations) by an optimization routine. Curve-fitting parameters included peak wavenumber, optical density at this wavenumber and (for those from the population neither aphakic nor constrained to ages where the standard lens transmissivity curve is supposed valid), density of the latter at the wavenumber of peak lens absorption. The average peak wavenumber of each population differed significantly from that of the other two. Either the standard curve of lens absorption (even with peak lens density as a curve-fitting parameter) is inappropriate for correcting the normal spectrum or the rhodopsins in the retinas of these populations do not all have identical wavenumbers of peak absorbance.
\end{abstract}

Human rhodopsin Rod vision Scotopic visibility

The preceding paper (Alpern et al, 1987) highlights a fundamental paradox in the study of rod vision. It is this. The action spectrum of human night vision [corrected for losses in the lens by the standard template of Wyszecki and Stiles (1967)] can be well matched to the spectral distribution of absorption coefficients of human rhodopsin measured in rod outer segments (ROS) with a microspectrophotometer (MSP) if the concentration-broadening mandated by the large peak specific absorbance $\left[\alpha\left(m_{0}\right) c=\right.$ $0.018 \log _{10}$ units $\mu \mathrm{m}^{-1}$, Bowmaker, 1981], also revealed by MSP of rhodopsin in mammalian ROS, is ignored (Bowmaker and Dartnall, 1980). Alternatively, essentially the same action spectrum corrected for losses in the lens with only a slightly denser version of the WyszeckiStiles template can also be matched to a rhodopsin absorption spectrum computed by "selfscreening' theory with a peak density $(0.35)$ consisted with MSP data using the spectral distribution of absorption coefficients obtained from the action spectrum of the photocurrent of single ROS (Macaca fascicularis) peaking at $m_{0}=20,400 \mathrm{~cm}^{-1}$ by ignoring the peak found by MSP $\left(m_{0}=19,996 \pm 64 \mathrm{~cm}^{-1}\right.$, Bowmaker et al., $1980 ; 19,900 \pm 78 \mathrm{~cm}^{-1}$, MacNichol et al., 1983) in this species (Baylor et al., 1984). It mitigates the contradiction in these observations only slightly, if at all, to add that the latter comparison assumes that the spectral distribution of the rhodopsin of human and $M$. fascicularis rods are identical despite the difference in the wavenumber of peak absorption revealed by MSP (for man $m_{0}=20,149 \pm 93 \mathrm{~cm}^{-1}$, Dartnall et al., 1983). In a word, essentially the same action spectrum, corrected for losses in the lens in nearly the same way, can be fitted either; (a) by ignoring the concentration-broadening required by the high specific absorbance found with MSP but utilising the MSP peak wavenumber $\left(m_{0}\right)$, or (b) by ignoring the MSP $m_{0}$ and utilizing the concentration-broadening required by the high specific absorbance revealed by MSP.

Although recognizing that it was inconsistent with the electrophysiology of ROS, Bowmaker and Dartnall (1980) introduced the suggestion that only the proximal-most ROS discs are physiologically active. In the preceding paper, Alpern et al., (1987) documented three lines of evidence inconsistent with any hypothesis leading to the inference that high peak rhodopsin density and the consequent "self-screening" is generally inappropriate for rod vision.

This paper reexamines factors often taken for granted in linking rhodopsin absorption with rod vision: the action spectrum of human rod vision, and it correction for losses in the lens by the standard curve. Specifically, it raises the possibility that the high peak density, the consequent self-screening and the peak wave- 
number of human rhodopsin measured in situ with MSP all might prove to be valid and appropriate for human rod vision provided individual differences are taken into consideration. Evidence from dichromats (Alpern and Pugh, 1977) and trichromats (Alpern and Moeller, 1977) suggesting that the absorption spectrum of the human long-wave sensitive cone pigment varies from one individual to the next, brings this possibility to mind.

Individual human rod vision action spectra were obtained from three sources. The first of these is the data of Crawford (1949). The second is $\Pi_{0}(\mu)$ field sensitivity action spectra measured in this laboratory over the last eight years or so. Finally, the individual rod action spectra of dark-adapted aphakic subjects-obtained by Tan (1971) in this laboratory-were analyzed.

\section{METHODS}

\section{(A) Subjects}

(1) C.I.E. scotopic curve. The C.I.E. scotopic visibility curve is the weighted average of 72 individual curves. Of these, 22 were measured at absolute threshold by Wald (1945) who published only the average of the group. Crawford (1949) obtained the other 50 spectra by suprathreshold brightness matching with a $20^{\circ}$ diameter bipartite photometric field. On one side was the reference "white" light, $3 \times 10^{-6} \mathrm{~cd} / \mathrm{ft}^{2}$; on the other was the monochromatic test. Data were obtained on young subjects less than 30 years of age in two experimental sessions: covering the spectral range $380-580 \mathrm{~nm}$ in the first, the range $480-780 \mathrm{~nm}$ in the second. Nine of the 50 subjects completed only one of the two sessions. To round out the group, Crawford added an additional nine subjects who completed only the other session. Since an emphasis of this paper is on individual differences, only the 41 observers ( 21 males, 20 females) completing both sessions are studied here. The data were converted to an equal quantum spectrum by multiplying the normalized visibilities (Table 3, Crawford, 1949) by the wavenumber.

(2) $\Pi_{0}(\mu)$ field sensitivity spectra. These were obtained in the course of other work; the spectra of four of the eight subjects have been published. For details of the procedure, readers are referred to these publications: Alpern and Kitahara (1983); Alpern et al. (1983); Abraham and Alpern (1984). In brief, the wavelength dependency of the radiance of a $5^{\circ}$ background was measured elevating the absolute threshold of a concentric $1^{\circ}$ blue-green $(500 \mathrm{~nm})$ test flash by a factor of ten.

(3) Aphakic subjects. Tan's (1971) research concerned vision in the ultra-violet, but he made measurements in the visible part of the spectrum up to $720 \mathrm{~nm}$. In the study of rod vision, he obtained the wavelength dependency of the absolute threshold of the dark-adapted retina $15^{\circ}$ temporal to the fovea for a $1^{\circ} \times 3^{\circ}$ vertical rectangular flash of light exposed for $0.2 \mathrm{sec}$ every $2 \mathrm{sec}$. Ten subjects varied in age between 15- and 70-years-old; they had lost their lenses for various reasons. None were suffering from eye disease. (For the mean of Tan's data, see Tan, 1971; Stark and Tan, 1982; Alpern and Pugh, 1974.)

\section{(B) Analysis}

Each of the 59 action spectra $[f(m)]$ plotted $\log f(m)$ as a function of wavenumber $(m)$ on a linear scale were fitted with the equation

$$
\begin{aligned}
& f(m)=A \tau_{1}^{\delta}(m) \tau_{2}(m) \\
& \times\left[1-10^{-\alpha(m) l}\right] /\left[1-10^{-\alpha\left(m_{0}\right) c l}\right]
\end{aligned}
$$

by a computer optimization routine STEPIT (Chandler, 1965) which searched the parameter space to select those free parameters which minimized the deviation of the measured data from that expected from equation (1). This approach, described by Pugh and Sigel (1978), has been slightly modified for the present purposes. $\alpha(m)$ is a standard template curve of fixed shape representing the spectral distribution of rhodopsin absorption coefficients. $c$ represents the concentration of rhodopsin in the rods and $l$, the rod outer segment length. $\alpha\left(m_{0}\right) c l$ represents the absorbance or optical density at the wavenumber of peak absorption, a free parameter used to optimize the computer fit to the measured action spectrum. No distinction is drawn in the analysis between $c$ and $l$ so that individual differences in peak density might be due to differences in $c$, differences in $l$, or to both. A second free parameter is $m_{0}$, the wavenumber of peak absorption. Variation of this parameter shifts the curve $\left[1-10^{-\alpha(m) c t}\right] /$ $\left[1-10^{-\alpha\left(m_{0}\right)<l}\right]$ horizontally. $A$ is a scale factor displacing the curve vertically as a third free parameter of the search routine.

(1) Losses in the eye media. $\tau_{1}^{\delta}(m)$ represents the spectral distribution of lens transmissivities. In the three groups they were analyzed differently. For aphakic subjects, it was assumed that $\delta=0$. For the subjects of Crawford's study, all of whom were of an age less than the age (30 
years) Crawford found the lens transmissivity began to show age-related changes, the standard spectral distribution of lens transmissivity $\tau_{1}(m)$ for human observers of this age group was used (Wyszecki and Stiles, 1967), i.e. $\delta$ was fixed at unity. The $\Pi_{0}(\mu)$ field sensitivity spectra were drawn from a population which included five observers older than this limit specified by Crawford to show age-related lens changes. Following Wyszecki and Stiles (1980), the final free parameter $\delta$ was also allowed to vary to help bring the r.m.s. of the deviations of the results for this group (and only this group) from the curve defined by equation (1) to a minimum. $\tau_{2}(m)$ was a small correction for losses in transmission through the remaining ocular media obtained from the spectrophotometric measurements of Boettner and Wolter (1962) on human eyes. This spectral distribution was the same for each spectrum.

(2) The nomogram $[\alpha(m)]$. Dartnall (1953) found that a standard shaped curve based on the absorption spectrum of frog rhodopsin plotted log absorption as a function of test wavenumber fitted similar plots of the absorption, and (ignoring losses due to short-wave absorbing photoproducts) the difference spectra, of rhodopsin of a variety of species by shifting the standard shape template on the wavenumber axis. However, the preceding paper showed that this generalization did not extrapolate well to mammalian rhodopsin and provided a more realistic curve for rat rhodopsin which agreed well (in a way the curve fitted with the Dartnall nomogram did not) with published data of the $m_{0}$ of rat rhodopsin. A similar procedure was followed here to fit the 59 human rod action spectra. The difference spectrum of the most recent measurements of human rhodopsin (Crescitelli, 1985) $440 \mathrm{~nm} \leqslant \lambda \leqslant 600 \mathrm{~nm}$ was used. This spectrum [plotted log absorbance difference as a function of wavenumber $\left(\mathrm{cm}^{-1}\right)$ ] was fitted by a fifth-order polynomial. To extrapolate beyond these limits on the short-wave extreme, the polynomial was equated with the most recent modification of the Dartnall nomogram (Wyszecki and Stiles, 1967). At the longwave extreme, the slope of the nomogram was gradually increased until it approached a straight line with a slope of $0.79 \mathrm{hv} / \mathrm{kT}=$ $1.59(10)^{-3}$ which Stiles (1948) found for the rod curve. A straight line on this plot is theoretically justified (Stiles, 1948), but its slope was arbitrarily selected and, to some extent, ad hoc. However, if it is not justified, it will have little effect on the results of this analysis which, for this and other reasons (cf. below) was not pressed in this part of the spectrum.

(3) Scale factor $A$. No analysis was made of individual differences in the scale factor $A$. The units were different in each of the three groups. Crawford's data were normalized at the peak wavelength, then quantized by multiplying by $m$. Tan's thresholds were in log (test) absolute sensitivity (log photons of the test $\left.\sec ^{-1} \mathrm{deg}^{-2}\right)^{-1}$; the $\Pi_{0}(\mu)$ spectra were in log field sensitivity (log photons of the background $\left.\sec ^{-1} \operatorname{deg}^{-2}\right)^{-1}$.

\section{(c) Intersession pooling}

In fitting the results for each subject from the two experimental sessions, Crawford found that the data at the five wavelengths which were obtained in both sessions did not link together perfectly. In deriving the final means, he took the data from the first session for wavelengths shorter than the peak wavelength $\left(\lambda_{0}\right)$ and those from the second session for wavelengths longer than $\lambda_{0}$. This example is not followed here. Instead, all available data for a given subject were pooled for the STEPIT fit of equation (1) to each action spectrum.

\section{RESULTS}

The result of applying the above routine to the mean of five repetitions of the $\Pi_{0}(\mu)$ field sensitivity spectrum of a single observer is shown by the smooth curve in Fig. 1 in which $\log$ field sensitivity (photons/s/ $\left./ \mathrm{deg}^{2}\right)^{-1}$ is plotted as a function of background field wavenumber. The root mean square deviation of experimental points from the fitted curve $(0.03663)$ approaches the precision of the radiometry of the light and of the calibration of the transmissivities of the filters. The free parameters which provide this fit are $m_{0}=20,544 \mathrm{~cm}^{-1}$, $\alpha\left(m_{0}\right) c l=1.295, \delta=1.723$, and $A=-4.207$. The STEPIT fit was then applied to each individual curve, the mean $( \pm 1 \sigma)$ of the r.m.s. deviations was slightly poorer, of course, $0.0426969 \pm 0.006482$, but the means of the five individual determinations of the free curvefitting parameters were remarkably close to those found for the average curve i.e. $m_{0}=$ $20,547 \pm 42.92 \mathrm{~cm}^{-1} \quad \alpha\left(m_{0}\right) c l=1.317 \pm 0.232$, $\delta=1.7248 \pm 0.0419$ and $A=-4.207 \pm 0.031$. A similar reliability was found when the same analysis was applied to the average of four 


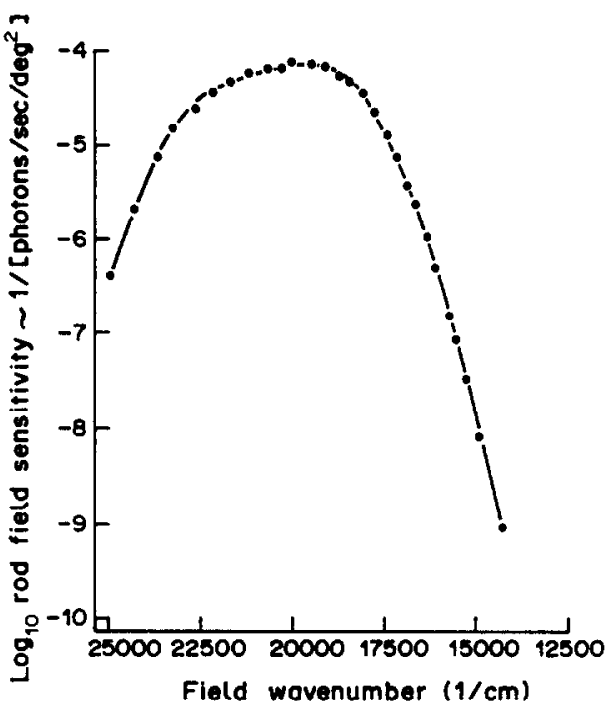

Fig. 1. Rod vision action spectrum operationally defined by $\Pi_{0}(\mu)$ field sensitivity for observer H.K. for a patch of retina $10^{\circ}$ temporal to the fovea. The ordinate is the $\log _{10}$ of the reciprocal of the field radiance (photons $\mathrm{sec}^{-1} \mathrm{deg}^{-2}$ ) elevating the threshold of a $500 \mathrm{~nm}$ monochromatic test by a factor of 10 above the absolute threshold. The abscissa values are represented in terms of the wavenumber (in $\mathrm{cm}^{-1}$ ) of the monochromatic backgrounds. The circular background was $5^{\circ}$ in diameter concentric with the $1^{\circ}$ $(200 \mathrm{msec})$ test, flashed once a second. The points are the means $\pm 1 \sigma(\mathrm{SD})$ of five experimental repetitions; the solid smooth curve is the solution to equation (1) found by the computer optimization routine STEPIT to minimize the r.m.s. deviation of the data from the curve. The r.m.s. deviation $=0.03663$.

repetitions of the measurements of $\Pi_{0}(\mu)$ field sensitivity on a second observer.

Some idea of the individual differences in $\Pi_{0}(\mu)$ field sensitivity can be gained from Fig. 2 which contains individual spectra of eight different observers. The points are empirical results, the curve is the best fit of equation (1) specified by the STEPIT routine. For each spectrum, the value of $m_{0}$ is designated by a small vertical line immediately above the curve. A fair difference (at the most, about $350 \mathrm{~cm}^{-1}$ ) in the value of $m_{0}$ is found between one observer and another. This is considerably larger than $3 \sigma$ of the five individual values of $m_{0}$ which go to make up the result in Fig. 1 for a single subject.

\section{Constraints}

Makous and Booth (1974) describe a cone-rod interaction under two-color increment threshold conditions not unlike those with which $\Pi_{0}(\mu)$ field sensitivity is measured. Makous and Peeples (1975) showed that as long as the background radiance was less than those elevating the absolute rod threshold by $1.5 \log _{10}$ units, no such interaction occurred. Since $\Pi_{0}(\mu)$ is defined as the radiance elevating absolute rod threshold $1.0 \mathrm{log}$ unit, there would appear to be no difficulty on this score. However, the nature of rod-cone interaction was not at all elucidated by Makous and Peeples, and the $1.5 \log$ unit dividing line was established by only a few experiments for backgrounds $\leqslant 630 \mathrm{~nm}$. Longer wave backgrounds of radiance required by the field sensitivity criterion would necessarily have a more powerful effect on cones relative to rods than those at $630 \mathrm{~nm}$, and the $1.5 \log _{10}$ unit dividing line might then be too high.

Similar problems plague the measurements of both Tan (1971) and Crawford (1949) in this part of the spectrum. In the former case, the absolute threshold was measured. It has been

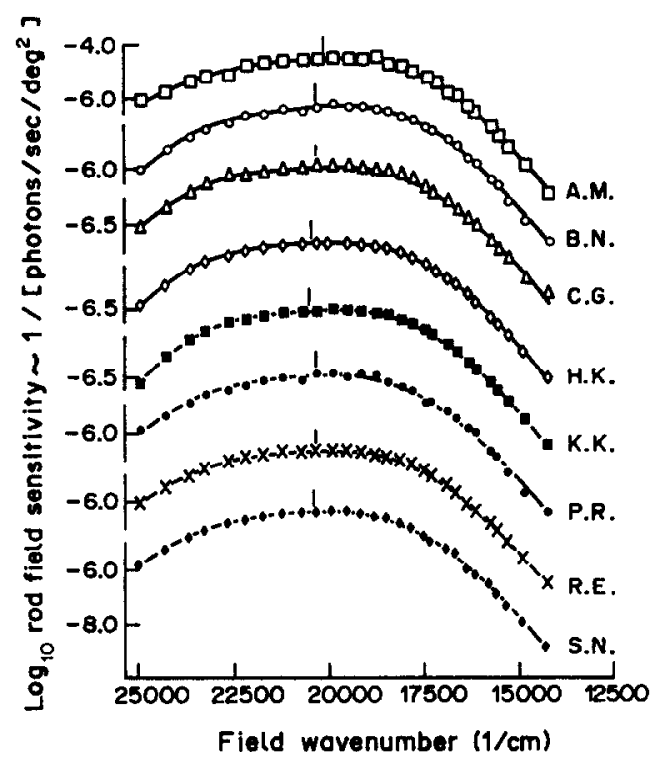

Fig. 2. Rod vision action spectra operationally defined by the $\Pi_{0}(\mu)$ field sensitivity of eight normal subjects. The ordinates and abscissae are the same as in Fig. 1, but the ordinate scale is compressed to display all eight spectra in one figure. The effect of this compression may be visualized by comparing the results for H.K. replotted here from Fig. 1 to the same data in that figure. For other details, see legend of Fig. 1. The value for $m_{0}$, the wavenumber of peak absorption of rhodopsin inferred by the STEPIT fit, is shown by the vertical line immediately above each spectrum. These have the following values: $20,235 \mathrm{~cm}^{-1}$ (A.V.), $20,459 \mathrm{~cm}^{-1} \quad$ (B.N.), $20,434 \mathrm{~cm}^{-1}$ (C.G.), $20,588 \mathrm{~cm}^{-1}$ (K.K.), $20,411 \mathrm{~cm}^{-1}$ (F.R.), 20,394 $\mathrm{cm}^{-1}$ (R.E.), and $20,448 \mathrm{~cm}^{-1}$ (S.N.). Note that the values in the preceding sentence differ from comparable data in the middle histogram of Fig. 3 because the curve fittings of the data of this figure (and Fig. 1) apply to all 28 data points while the analysis which Figs 3 and 4 summarize were obtained after the results at the six lowest wavenumbers were omitted in order to meet the curve-fitting constraints discussed in the text. 
recognized, at least since Stiles (1939), that the dark-adapted absolute threshold for some parts of the extrafoveal human retina for monochromatic lights of wavelengths longer than about $620 \mathrm{~nm}$ may be influenced by cones. Crawford and Palmer (1985) have now studied interaction in heterochromatic brightness matching under the conditions of Crawford's (1949) measurements as well as at absolute threshold in the peripheral retina. They found in brightness matching a mixture of red and green lights to be more effective than either by itself (i.e. superadditivity); at threshold they found the opposite (subadditivity). This led them to infer that the "... scotopic visibility curve cannot therefore represent a linear response from only one class of retinal receptors especially in the long-wave part of the spectrum." Though they equivocate on defining the spectral limits representing a pure rod response under the conditions Crawford's data were obtained, their results suggest the longest possible wavelength to be in the orange $(\lambda \sim 620 \mathrm{~nm})$.

For the above reasons, the statistical analysis which follows concerns 22 wavelengths in the spectral range $400-610 \mathrm{~nm}$ in the case of the $\Pi_{0}(\mu)$ field sensitivity spectra, 22 wavelengths in spectral range $400-620 \mathrm{~nm}$ for the aphakic spectra, and 17 data points at 12 different wavelengths in the spectral range $400-620 \mathrm{~nm}$ for the scotopic visibility curves measured by Crawford (1949).

Individual differences in the peak wavenumber $\left(m_{0}\right)$ of the action spectrum of human rhodopsin

It was expected that the distribution of $m_{0}$ from the 59 spectra would form an homogeneous group well fit by the bell-shaped normal curve $\left(\bar{m}_{0} \pm 1 \sigma=20,118.61 \mathrm{~cm}^{-1} \pm 176.69 \mathrm{~cm}^{-1}\right)$. In fact, this distribution is well fitted by that curve. [The measure $\left(g_{1}\right)$ of skewness of such a curve is 0.2646; its measure of kurtosis $g_{2}=-0.2386$. But the group is not homogeneous! A one-way analysis of variance of the three subsets shows that the means of each: $19,918 \pm 119.34 \mathrm{~cm}^{-1}$ (aphakic observers), $20,111.41 \pm 109.79 \mathrm{~cm}^{-1}$ (scotopic visibility) and $20,280.88 \pm 228.88 \mathrm{~cm}^{-1}\left[\Pi_{0}(\mu)\right.$ spectra] differs significantly from the others, $F(2,56)=15.55$, $P<0.001$.

The histograms in Figs 3 and 4 are consequently shown as separate distributions for each subset. Although the distribution of $m_{0}$ of this entire population is well fitted by a normal bell-shaped curve, for none of the subpopu-
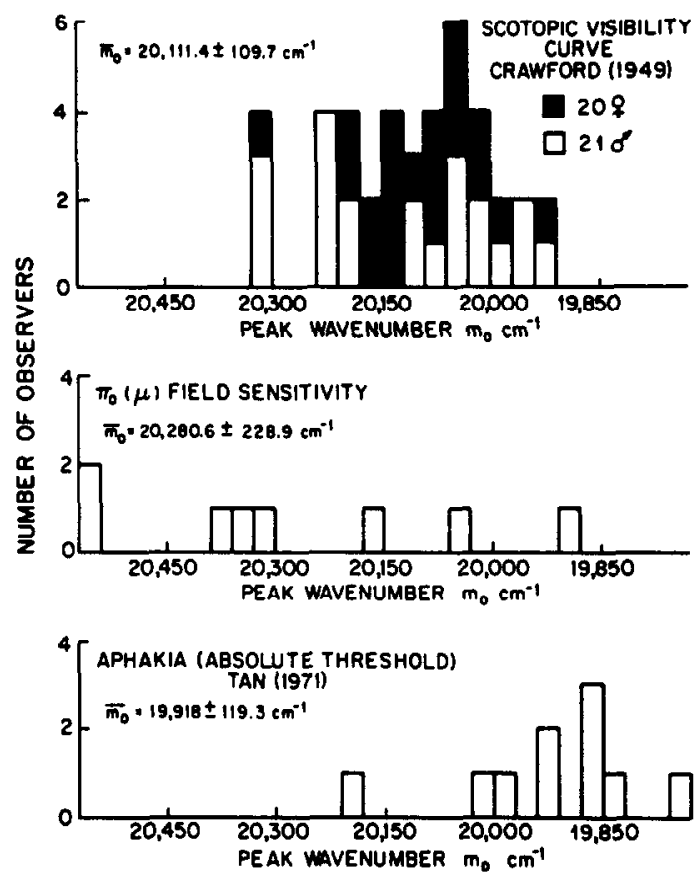

Fig. 3. Frequency distribution of peak wavenumber $\left(m_{0}\right)$ of human rhodopsin obtained by the computer optimization routine to each of the 59 action spectra anlyzed in this study. Top: 20 females (solid rectangles) and 21 males (open rectangles) analyzed by Crawford (1949). Middle: three females and five males $\Pi_{0}(\mu)$ field sensitivity action spectra (no sex discrimination is represented in this histogram). Bottom: absolute threshold action spectra obtained by Tan (1971) on 10 male aphakic observers.

lation histograms is this true. The distribution of $m_{0}$ of the curves from Crawford's study (top, Fig. 3 ) is not particularly skewed $\left(g_{1}=0.2955\right)$, but it is flat (i.e. platykurtic). Its measure of kurtosis $\left(g_{2}=0.7460\right)$ is three times larger than the comparable value for the distribution curve of $m_{0}$ for the entire group. The same features characterized the distribution curve of $m_{0}$ for $\Pi_{0}(\mu)$ (middle histogram, Fig. 3) though the small sample size makes the interpretation less clear. The value for $g_{1}=-0.3616$ perhaps suggests slight skewed distribution to the left (i.e. high-frequency end of the spectrum); the measure of kurtosis $g_{2}=-0.9821$ ) is more than four times larger than that of the entire sample. On the other hand, the histogram of the distribution of $m_{0}$ threshold in aphakia (lowermost histogram in Fig. 3) is not kurtotic; its measure of kurtosis $\left(g_{2}=0.2027\right)$ is even closer to zero than is the case of the full population distribution, but it is skewed to the low frequency side of the spectrum. The measurement of skewness $\left(g_{1}=0.9945\right)$ is $3.75 \times$ the value obtained for the population histogram of $m_{0}$ values.

While the reason for this skewed distribution requires further work on a larger aphakic pop- 


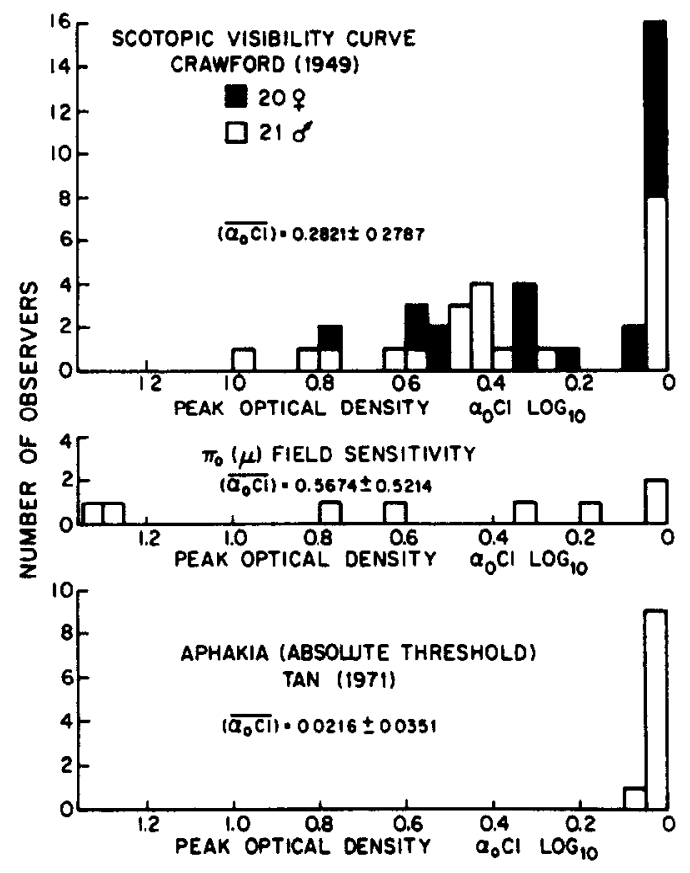

Fig. 4. Frequency distribution of the common log optical density at the wavenumber of maximum absorption $\left[\alpha\left(m_{0}\right) c l\right]$ of human rhodopsin inferred from the computer optimization routine fits to the 59 spectra analyzed in this study. The arrangement of this figure: [scotopic visibility data of Crawford (1949) at the top, the $\Pi_{0}(\mu)$ field sensitivity data in the middle, and the aphakic eyes absolute threhsolds below]. The symbols are those used in Fig. 3 to the legend of which readers are referred for further details.

lation, the fact of it is partly responsible for the statistically significant difference between the average $m_{0}$ for the aphakic observers and that of Crawford's population. On the other hand, the significant difference between the mean $m_{0}$ of the $\Pi_{0}$ field sensitivity spectra and the mean of Crawford's data has a different cause, discussed below.

Before turning to this, consider the possibility that the use of STEPIT itself introduced a bias which distorts the estimates of $m_{0}, \alpha\left(m_{0}\right) c l$ or of $\delta$. Three ideal rhodopsin action spectra simulating the conditions (i.e. the number of wavelengths and the parts of the spectrum experimentally examined) of the three subsets of this sample were calculated, assuming the parameters $m_{0}=20,000 \mathrm{~cm}^{-1}, \alpha\left(m_{0}\right) c l=0.5$, and $\delta=1.0$ together with an appropriate scale factor A. Each of these spectra were in turn parametrically perturbed and fitted as if it were an actual spectrum. The amount of random perturbation was chosen so that the best fit provided by STEPIT to each perturbed spectrum had approximately the same root mean square (r.m.s.) deviations from the fit by eqution
(1) as the subset simulated. This procedure, known as parametric bootstrapping (Efron, 1982), was repeated 100 times for each subset. The actual values (mean $\pm 1 \sigma$ ) for the three simulated subsets of Crawford, aphakia and $\Pi_{0}(\mu)$ were respectively for $\bar{m}_{0}: 20,000.66 \pm$ $57.44 \mathrm{~cm}^{-1}, \quad 20,000.51 \pm 57.42 \mathrm{~cm}^{-1}, \quad$ and $20,005.82 \pm 61.56 \mathrm{~cm}^{-1}$; for $\overline{\alpha\left(m_{0}\right) c l}: 0.5192 \pm$ $0.2533,0.491 \pm 0.1736$ and $0.5191 \pm 0.1436$. For $\delta$ only the subset simulating $\Pi_{0}(\mu)$ field sensitivity was evaluated; the result was $\delta=1.0021 \pm 0.0704$. There is no suggestion that the use of the STEPIT routine introduced any measurable bias in the estimation of $m_{0}, \alpha\left(m_{0}\right) c l$ or $\delta$.

\section{Individual differences in peak density $\left[\alpha\left(m_{0}\right) c l\right]$ of rhodopsin in vivo}

The comparable histograms of the distributions of the peak densities of the three subsets of the 59 spectra are shown in Fig. 4. The mode of each subset is in the smallest density interval $(0.0-0.049)$. For two of the three subsets (the exception is the distribution of peak densities among aphakic subjects), there is a suggestion of bimodal distribution of peak densities. The meaning of this trend is by no means clear. The mean peak density of rhodopsin inferred from the fits to Crawford data was $0.2822 \pm 0.2787$; for the fit to the $\Pi_{0}(\mu)$ spectra, it was $0.5674 \pm 0.5214$; for the aphakia results, $0.0216 \pm 0.0351$. In view of the findings of the preceding paper, these results suggest that for a substantial minority of the population-say, for example, for the subset of 18 subjects with peak densities less than 0.2 in Crawford's contribution to the C.I.E. visibility curve-Bowmaker and Dartnall (1980) accurately illuminated the relation between the rhodopsin concentration in their rods and their scotopic visibility curves. Closer examination of the details reveals however that even here the matter is more complex. The mean wavenumber of peak absorbance of the rhodopsin in the rods of the subjects in this subset is $20,021.6 \pm 58.59 \mathrm{~cm}^{-1}$ or $127.4 \mathrm{~cm}^{-1}$ smaller than the mean peak in the most recent MSP of 39 rods from 7 human eyes (Dartnall $e t$ al., 1983). This difference is highly significant statistically $(t=5.36,55 \mathrm{df}, P<0.001)$.

\section{Differences in lens density}

The mean of the value for $\delta=1.4024 \pm 0.375$ $\left[\Pi_{0}(\mu)\right.$ spectra] is appreciably higher than unity. This is due to two factors: (i) the majority of this subset were of an age above the borderline 
Crawford (1949) found deviations (in the shortwave part of the spectrum) from the standard lens transmissivity spectrum curves first began to appear; (ii) three of the five older subjects were oriental observers. Increased absorbance of light from the short-wave extreme of the spectrum in one of these eyes was reported by Alpern and Kitahara (1983); the present results suggest it may be a more general characteristic of such eyes. Indeed, the three highest values of $\delta$ were obtained from these observers and their mean value $\delta=1.7643 \pm 0.2564$ is statistically significantly larger $(t=3.25,6 d . f ., P<0.02)$ than that for the five other observers $\delta=1.1854 \pm 0.2378$, despite the small sample size.

\section{DISCUSSION}

These results suggest that either the inappropriateness of the standard Wyszecki and Stiles lens template or the assumption that all human rhodopsins have identical wavenumbers of peak absorption, or both of these, could be repsonsible for the failure to match the C.I.E. scotopic curve with the absorption spectrum inferred from MSP data.

In the analysis of Crawford's data, it was assumed that the spectral transmissions of these 41 eyes were identical and that individual differences were due to individual differences in rhodopsin. Norren and Vos (1974) did exactly the opposite, i.e. assumed rhodopsin had the identical absorption spectrum and that all the differences were due to differences in the spectral transmissivity of the lens. This is certainly the more conventional view. The lens template they derive, however, is incorrect because the wavelength of peak rhodopsin absorption used is inconsistent with MSP data (Bowmaker and Dartnall, 1980; Dartnall et al., 1983). Each of these approaches may well prove an unrealistic extreme, and careful readers will wonder why $\delta$ was not allowed to vary in the optimization routine fit of equation (1) to Crawford's data. The answer is found in the wavelength constraints of the analysis which so reduced the number of experimental points available in the data that only three wavelengths were available for curve fitting for each curve-fitting parameter. This small ratio occasionally resulted in quite unphysiological parameters (e.g. a peak optical density of 3.26 , implying an outer segment about as long as $45 \%$ of the distance between the outer edge of the pigment epi- thelium and the internal limiting membrane). Such ambiguities never resulted from the analysis used in this paper where this ratio was $4 / 1$ for Crawford's data and $5.5 / 1$ for the $\Pi_{0}(\mu)$ field sensitivity spectra. Of the two extremes, given Crawford's observation that his subjects were young enough to exclude the changes in lens absorption due to age, it was decided to explore the possibility that $m_{0}$ varied by fixing $\delta=1.0$ (though, without doubt, it is a simplification). But it may be mentioned that when $m_{0}$ and $\delta$ are both varied as free parameters, the results offer no comfort to those who maintain that the peak wavenumbers of rhodopsin in Crawford's subjects were identical. The curve fitting greatly reduces the r.m.s. error of the fit of each spectrum, but the variance of both lens absorption and wavenumber of peak rhodopsin absorption in this population remains substantial. For example, the variance in peak wavenumber $(21,668)$ is even larger than that variance $(12,056)$ resulting from the analysis of when $\delta$ is fixed at unity illustrated by the distribution at the top of Fig. 3.

It was noted that $\delta$ was smaller in the five occidental eyes than in the three oriental eyes of the $\Pi_{0}(\mu)$ group. If the lens template is inappropriate for either subset, then perhaps the statistically significant $(t=2.83,6$ d.f., $P<0.05)$ differences between them in $m_{0}$ is also explained. The mean of the former $\left(20,155.2 \pm 181.6 \mathrm{~cm}^{-1}\right)$ is substantially smaller than that of the latter $\left(20,489.7 \pm 111.9 \mathrm{~cm}^{-1}\right)$ subset but not $(t=0.78,44 d . f$. ) from the mean peak wavenumber $\left(20,111.4 \pm 109.8 \mathrm{~cm}^{-1}\right)$ of rhodopsin inferred from Crawford's data. This last provides further justification for fixing $\delta=1.0$ in fitting Crawford's data, but allowing $\delta$ to vary in fitting the $\Pi_{0}(\mu)$ spectra.

Though the use of an inappropriate lens template might well account for all the variability found in the action spectrum of rod vision, the free curve-fitting parameter $\delta$ allowed the range of lens absorption spectra available in the literature access to the curve-fitting process. Hence, modification of the standard template which might allow a single free parameter to bring the two extremes $(20,564$ and $19,768 \mathrm{~cm}^{-1}$ ), together with all the other peak wavenumbers in the histograms in Fig. 3, into coincidence while remaining consistent with published lens absorption data, are neither trivial nor easy to formulate.

So it may prove fruitful to entertain the possibility that wavenumber of peak rhodopsin 
absorption differs in normal human eyes just as the peak wavenumbers of human erythrolabe (Alpern and Pugh, 1977; Alpern and Moeller, 1977; Alpern, 1986) and of frog rhodopsin (Bowmaker et al., 1975) seem to do. Variability of rhodopsin peak wavenumber among different individuals in the same subset is large in the present study, and there is evidence to suggest that it cannot all be attributed to measurement imprecision. In the $\Pi_{0}(\mu)$ subset, the variance of the peak wavenumber of rhodopsin inferred from the experimental population was 13.82 times larger than the variance of the peak wavenumber of rhodopsin inferred from the bootstrap simulated population; for Crawford's data it was 3.65 , and for the aphakia subset it was 4.32. All these $F$ values are significantly different from zero $(P<0.01, P<0.001$, $0.01<P<0.05$, respectively).

If human rhodopsin, like erythrolabe and frog rhodopsin, may have different peak wavenumbers in different normal eye, studies like Bowmaker and Dartnall's, in which the MSP $m_{0}$ of rhodopsin from one normal eye is compared with the $m_{0}$ of the average action spectrum of 72 different normal eyes, will be of considerably less analytical interest than comparisons of action spectrum and MSP peak wavenumber of rhodopsin from the same eye. If different populations are to be drawn for the MSP and action spectrum samples, at the very least MSP $m_{0}$ of normal thodopsin from a much larger sample of normal eyes is required. More recently, Dartnall et al. (1983) report results of MSP study of rods from five more human retinas. They found rhodopsin with mean peak wavenumbers which varied in different eyes from $20,243 \mathrm{~cm}^{-1}$ (one rod) to $20,113 \mathrm{~cm}^{-1}$ (five rods). The average of 39 rods from these six eyes was $20,149 \pm 93 \mathrm{~cm}^{-1}$.

This mean value is in good agreement with the mean $m_{0}$ inferred for rhodopsin from Crawford's data. But the platykurtic distribution at the top of Fig. 3 [the usual kurtosis statistic (Snedecor and Cochran, 1967) $g_{2}=-0.7460$; Geary's kurtosis statistic $a=0.8382$ ] is otherwise quite unlike the leptokurtic $\left(g_{2}=+0.8799\right.$, $a=0.7537$ ) MSP distribution. If both histograms reflect the identical visual pigment of fixed peak wavenumber, each randomly perturbed by (different) measurement imprecisions, both should have the bell shape of the normal probability distribution. But the observed differences in kurtosis of these two histograms would not be surprising if the $m_{0}$ of rhodopsin in different normal eyes differed, since the psychophysical data were obtained on 41 normal eyes while the MSP data come from only six. The evidence is suggestive rather than compelling since the kurtosis of neither distribution can be shown to differ from the normal curve at the 0.05 level of significance. [Tables for the $g_{2}$ statistic are generally not available for sample sizes less than 50; the $a$ of the MSP data gives $0.05<P<0.1$; for Crawford's data $P>0.1$ $(P=0.1$, for $\mathrm{a}=0.8436)]$. There is no other standard way of testing the statistical significance of the difference in shapes of two such curves, one clearly platykurtic, the other obviously leptokurtic. A more compelling inference may emerge from a study comparing results from larger populations.

Another test of the view that the peak wavenumber of rhodopsin of different normal eyes differ would be to compare mean $m_{0}$ inferred from rod action spectra measured on different individual observers once the measurements have been repeated enough times that reasonable estimates of each observer's measurement error is available. This is a more formidable task; so far it has only been completed on the observer whose $\Pi_{0}(\mu)$ field sensitivity results are shown in Fig. $1(n=5)$ and on his older brother $(n=4)$. The mean $\delta$ for the former was $1.60 \pm 0.07$; for the latter it was $2.06 \pm 0.14$. This difference is statistically significant $(t=6.50,7$ d.f. $P<0.001)$ and no doubt is due to the six year age difference between the observers. The mean peak wavenumber for the observer in Fig. $1\left(\bar{m}_{0}=20,367 \pm 70.49\right)$ differs significantly $(t=2.79,7 \mathrm{~d}, \mathrm{f}$., $0.05>P>0.02)$ from the values inferred from his brother's $\Pi_{0}(\mu)$ spectra $\left(\bar{m}_{0}=20,578.5 \pm 151.96\right)$, while the mean peak densities of the rhodopsin inferred from the $\Pi_{0}(\mu)$ field sensitivities of the two observers do not differ significantly $(t=2.24,0.1>P>0.05)$. These results are consistent with different peak wavenumbers of the rhodopsin in different normal eyes, even when the two observers are siblings.

Individual differences in the peak wavenumber of rhodopsin absorption would, of course, account for the significant difference between $m_{0}$ inferred from rod action spectra measured on oriental and occidental eyes. What is not so clear is how to account for the significant difference between the mean peak wavenumber of rhodopsin inferred from the aphakic action spectra and the value inferred from Crawford's data measured on eyes with 
normal lenses. The operations which defined these two sets of spectra were, of course, different. If the precautions taken above to avoid the cone-rod interaction described by Crawford and Palmer (1985) were ineffective in obviating substantial effects of this kind, the results might be accounted for since they found superadditivity under the conditions Crawford's spectra were measured and subadditivity under the conditions which defined the aphakic action spectra. The comparison of $m_{0}$ inferred from rod action spectra measured on normal and aphakic eyes by identical procedures should clarify this possibility.

\section{SUMMARY}

1. Fifty-nine action spectra of human rod vision, each from a different subject from one of three populations, were analyzed by fitting a template for the spectral distribution of human rhodopsin absorption coefficients by a computer optimization routine. The free curve-fitting parameters were wavenumbers of peak absorption, optical density at this wavelength and (for the population neither aphakic nor within the age range for which the corrections for spectral losses in the human lens are thought to be defined by the standard spectral distribution of lens absorption of Wyszecki and Stiles, 1967) the peak density of the absorption spectrum of the human lens.

2. Although the distribution of peak wavenumbers in this population is well fit by a normal probability curve, the mean peak wavenumber of each subpoplation differs significantly from that of the other two.

3. This result can be explained either by assuming that the Wyszecki and Stiles template for lens absorption is grossly erroneous or that pronounced individual differences in the spectral distribution of the absorption coefficients of human rhodopsin exist.

4. Either explanation could account for failure of Bowmaker and Dartnall (1980) to fit the human scotopic visibility function with the absorption spectrum of human rhodopsin inferred from the microspectrophotometry of single rod outer segments of a human retina.

Acknowledgements-Assisted by Research Grant EY-00197 from the National Eye Institute. I am grateful to K. E. W. P. Tan, M.D., for permission to include his results in this analysis. I thank Austra Liepa for programming asssistance and Professor Lawrence Maloney for suggesting the parametric bootstrap simulation.

\section{REFERENCES}

Abraham F. A. and Alpern M. (1984) Factors influencing threshold of the fundamental electrical response to sinusoidal excitation of human photoreceptors. J. Physiol. Lond. 357, 151-172.

Alpern M. (1987) Variation in the visual pigment of human dichromats and normal human trichromats. Frontiers of Visual Science, pp. 169-193. National Academy Press, Washington, D.C.

Alpern M. and Kitahara K. (1983) The directional sensitivity of the Stiles' colour mechanisms. J. Physiol., Lond. 338, 651-668.

Alpern M. and Moeller J. (1977) The red and green cone visual pigments of deuteranomalous trichromacy. J. Physiol., Lond. 266, 647-675.

Alpern M. and Pugh E. N. Jr (1974) The density and photosensitivity of rhodopsin in the living retina. J. Physiol., Lond. 237, 341-370.

Alpern M. and Pugh E. N. Jr (1977) Variation in the action spectrum of erythrolabe among deuteranopes. J. Physiol., Lond. 266, 613-646.

Alpern M., Ching C. C. and Kitahara K. (1983) The directional sensitivity of retinal rods. J. Physiol. 343, 577-592.

Alpern M., Fulton A. B. and Baker B. N. (1987) "Selfscreening" of rhodopsin in rod outer segments. Vision Res. 27, 1459-1480.

Baylor D. A., Nunn B. J. and Schnapf J. L. (1984) The photocurrent noise and spectral sensitivity of rods of the monkey Macaca fascicularis. J. Physiol., Lond. 357, 575-607.

Boettner E. A. and Wolter J. R. (1962) Transmission of ocular media. Invest. Ophthal. 1, 776-783.

Bowmaker J. K. (1981) Visual pigments and colour vision in man and monkeys. J. R. Soc. Med. 74, 348-356.

Bowmaker J. K. and Dartnall H. J. A. (1980) Visual pigments of rods and cones in a human retina. J. Physiol. 298, 501-511.

Bowmaker J. K., Dartnall H. J. A. and Mollon J. D. (1980) Microspectrophotometric demonstration of four classes of photoreceptor in an Old World primate. Macaca fascicularis. J. Physiol. 298, 131-143.

Bowmaker J. K., Loew E. R. and Liebman P. A. (1975) Variation in the $\lambda_{\max }$ of rhodopsin from individual frogs. Vision Res. 15, 997-1003.

Chandler J. P. (1965) STEPIT. Quantum chemistry exchange program. Indiana Univ. Bloomington, Ind.

Crawford B. H. (1949) The scotopic visibility curve. Proc. Phys. Soc. 62B, 321-334.

Crawford B. H. and Palmer D. A. (1985) The scotopic visibility curve and cone intrusion. Vision Res. 25, 863-866.

Crescitelli F. (1985) Some properties of solubilized human rhodopsin. Exp. Eye Res. 40, 521-535.

Dartnall H. J. A. (1953) The interpretation of spectral sensitivity curves. Br. Med. Bull. 9, 24-30.

Dartnall H. J. A., Bowmaker J. K. and Mollon J. D. (1983) Human visual pigments: microspectrophotometric results from the eyes of seven persons. Proc. R. Soc. 220B, 115-130.

Efron B. (1982) The Jackknife, the Bootstrap and Other Resampling Plans, pp. 27-36. Soc. Industr. Appl Math Monogr.

MacNichol E. F. Jr, Levine J. S., Mansfield R. J. W., Lipetz 
L. E. and Collins B. A. (1983) Microspectrophotometry of visual pigments in primate photoreceptors. In Colour Vision, Physiology and Psychophysics (Edited by Mollon J. D. and Sharpe L. T.), pp. 13-38. Academic Press, New York.

Makous W. and Boothe R. (1974) Cones block signals from rods. Vision Res. 14, 285-294.

Makous W. and Peeples D. (1979) Rod-cone interaction: reconciliation with Flamant and Stiles. Vision Res. 19, 695-698.

Norren D. V. and Vos J. J. (1974) Spectral transmission of the lens. Vision Res. 14, 1237-1244.

Pugh E. N. Jr and Sigel C. (1978) Evaluation of the candidacy of the $\Pi$-mechanisms of Stiles for colormatching fundamentals. Vision Res. 18, 317-330.

Snedecor G. W. and Cochran W. G. (1977) Statistical Methods, 6th edn, pp. 86-89. Iowa State Univ. Press, Ames, Iowa.

Stark W. S. and Tan K. E. W. P. (1982) Ultraviolet light: photosensitivity and other effects on the visual system. Photochem. Photobiol. 36, 371-380.

Stiles W. S. (1939) The directional sensitivity of the retina and the spectral sensitivities of the rods and cones. Proc. R. Soc. 127B, 64-105.

Stiles W. S. (1948) The physical interpretation of the spectral sensitivity curve of the eye. Trans. Opt. Conv. Worshipful Co. Spectacle Makers, pp. 97-107. Spectacle Makers' Co. London.

Tan K. E. W. P. (1971) Vision in the ultraviolet. Thesis, Univ. of Utrecht, Drukkerij Elinkwijk, Utrecht.

Wald G. (1945) Human vision and the spectrum. Science, N.Y. 101, 653-658.

Wyszecki G. and Stiles W. S. (1967) Color Science: Concepts and Methods, Quantitative Data and Formulas, p. 217. Wiley, New York.

Wyszecki G. and Stiles W. S. (1980) High-level trichromatic color matching and the bleaching hypothesis. Vision Res. 20, 23-37. 